NASZA DERMATOLOGIA Online OUR DERMATOLOGY Online

Source of Support: Nil

Competing Interests: None

\section{A STUDY ON DERMATOSES OF PREGNANCY}

\author{
Neerja Puri, Asha Puri \\ Department of Dermatology and Venereology, Punjab Health Systems Corporation, \\ Ferozepur, Punjab, India \\ Corresponding author: Dr. Neerja Puri \\ neerjaashu@rediffmail.com
}

\begin{abstract}
Certain dermatoses are specifically seen in pregnancy or postpartum period. It is therefore important for the clinicians to recognize and treat these cutaneous disorders to minimize maternal and fetal morbidity. The commonest pregnancy related dermatoses was polymorphic eruption of pregnancy seen in $22 \%$ patients, prurigo of pregnancy was seen in $7 \%$ patients, pemphigoid gestationis was seen in $3 \%$ patients, pruritic folliculitis of pregnancy was seen in $2 \%$ patients and intrahepatic cholestasis was seen in $1 \%$ of patients. It was seen that the skin disorders were commonest in the third trimester $(60 \%)$, followed by $31 \%$ patients in second trimester and $9 \%$ patients in first trimester.
\end{abstract}

Key words: pregnancy; dermatoses; prurigo; pruritis; urticaria; polymorphic eruption

\section{Introduction}

Pregnancy is a time of immense hormonal, immunologic, and vascular changes [1]. Whether physiologic or pathologic, these changes affect virtually every organ of the pregnant woman, including the skin [2]. Indeed, certain dermatoses are seen almost exclusively during pregnancy or the postpartum period. Hence, awareness and recognition of these conditions and familiarity with their treatment and outcomes are important [3]. These conditions can be divided into three main categories: physiologic changes associated with pregnancy; preexisting dermatoses affected by pregnancy; and dermatoses specific to pregnancy. This paper concentrates on the dermatoses specific to pregnancy, common dermatological disorders asspociated with pregnancy and sexually transmitted diseases of pregnancy. Although the dermatoses of pregnancy are believed to be a direct result of gestation or the products of conception, they are classified as pathologic processes [4]. The main diseases under this heading include pruritic urticarial papules and plaques of pregnancy, pemphigoid gestationis, intrahepatic cholestasis of pregnancy, pruritic folliculitis of pregnancy, and prurigo of pregnancy [5].

\section{Material and Methods}

The study was conducted in the dermatology out-patient department with referral cases coming from obstetrics and gynecology OPD. Ethical committee clearance was obtained. Written informed consent was taken from all the patients before thev study. Detailed history including demographic data, chief complaints related to skin, presence of itching, skin lesions, onset in relation to duration of pregnancy, jaundice, vaginal discharge, past or family history of similar lesions, exacerbating factors, associated medical or skin disorders etc. was elicited and recorded. Complete cutaneous examination was done in all cases to study all the physiological changes of skin and its appendages. If any specific dermatosis of pregnancy was present, the morphology of skin lesions, distribution and the sites involved were studied. Relevant systemic examination was carried out. If any preexisting skin disease was present, any evidence of exacerbation or remission was recorded. Appropriate investigations were done to confirm diagnosis if required. Bedside laboratory procedures like Tzanck smear, KOH mount and Gram's stain were carried out. To confirm diagnosis skin biopsy and DIF were done in a few cases. In all cases with history of pruritus related to specific disorders of pregnancy, liver function tests were done. Screening with VDRL and ELISA for HIV was done in all the cases. Examination of the ,contact' was done in all cases of sexually transmitted disease. Results were tabulated and analyzed.

\section{Results}

The commonest pregnancy related dermatoses was polymorphic eruption of pregnancy seen in $22 \%$ patients, prurigo of pregnancy was seen in $7 \%$ patients, pemphigoid gestationis was seen in 3\% patients, pruritic folliculitis of pregnancy was seen in $2 \%$ patients and intrahepatic cholestasis was seen in $1 \%$ of patients (Tabl. I). 


\begin{tabular}{|l|l|l|l|}
\hline \multicolumn{1}{|c|}{ Sr No } & \multicolumn{1}{|c|}{ Specific pregnancy dermatoses } & Number & \multicolumn{1}{c|}{ Percentage (\%) } \\
\hline 1 & Polymorphic Eruption Pregnancy & 22 & 22 \\
\hline 2 & Pemphigoid gestationis & 3 & 3 \\
\hline 3 & Prurigo of pregnancy & 7 & 7 \\
\hline 4 & Pruritic folliculitis of pregnancy & 2 & 2 \\
\hline 5 & Intrahepatic cholestasis of pregnancy & 1 & 1 \\
\hline
\end{tabular}

The commonest associated dermatological disorder in pregnancy was striae distensae (62\%), 16\% patients had scabies and $15 \%$ patients had superficial fungal infections.
Melasma was seen in $14 \%$ patients, candidiasis in $12 \%$ patients, gingivitis in $6 \%$ patients, acne in $5 \%$ patients and urticaria was seen in $4 \%$ patients (Tabl. II).

\begin{tabular}{|l|l|l|l|}
\hline \multicolumn{1}{|c|}{ Sr No } & \multicolumn{1}{|c|}{ Dermatological disorder } & Number & Percentage (\%) \\
\hline 1 & Scabies & 16 & 16 \\
\hline 2 & Superficial fungal infections & 15 & 15 \\
\hline 3 & Candidiasis & 12 & 12 \\
\hline 4 & Striae distensae & 62 & 62 \\
\hline 5 & Urticaria & 4 & 4 \\
\hline 6 & Melasma & 14 & 14 \\
\hline 7 & Gingivitis & 6 & 6 \\
\hline 8 & Acne & 5 & 5 \\
\hline
\end{tabular}

Table II. Asociated dermatological disorders in pregnancy

From the above table, it is clear that the commonest sexually transmited disease in pregnancy was discharge per vaginum seen in $28 \%$ patients, genital warts were seen in 65 patients, molluscum contagiosum was seen in 5\% patients and syphilis was seen in $4 \%$ patients (Tabl. III).

\begin{tabular}{|l|l|l|l|}
\hline \multicolumn{1}{|c|}{ Sr No } & \multicolumn{1}{c|}{ Sexually Transmited Disease } & Number & Percentage (\%) \\
\hline 1 & Syphilis & 4 & 4 \\
\hline 2 & Genital warts & 6 & 6 \\
\hline 3 & Molluscum contagiosum & 5 & 5 \\
\hline 4 & Discharge per vaginum & 28 & 28 \\
\hline
\end{tabular}

Table III. Sexually transmitted diseases in pregnancy

\section{Discussion}

In our study, it was seen that the commonest age group affected was between 21-30 years of age followed by $30 \%$ patients in the age group of $31-40$ years of age; $15 \%$ patients were between 11-20 years of age and $10 \%$ patients were more than 40 years of age. There were $40 \%$ primigravida and $60 \%$ multigravida in our study. The commonest pregnancy related dermatoses was polymorphic eruption of pregnancy seen in $22 \%$ patients, prurigo of pregnancy was seen in $7 \%$ patients, pemphigoid gestationis was seen in 3\% patients, pruritic folliculitis of pregnancy was seen in $2 \%$ patients (Fig. 1) and intrahepatic cholestasis was seen in $1 \%$ of patients. It was seen that the skin disorders were commonest in the third trimester $(60 \%)$, followed by $31 \%$ patients in second trimester and $9 \%$ patients in first trimester. The commonest associated dermatological disorder in pregnancy was striae distensae (62\%), $16 \%$ patients had scabies and $15 \%$ patients had superficial fungal infections (Fig. 2). Melasma was seen in $14 \%$ patients, candidiasis in $12 \%$ patients, gingivitis in $6 \%$ patients, acne in $5 \%$ patients and urticaria was seen in $4 \%$ patients. The commonest sexually transmited disease in pregnancy was discharge per vaginum seen in $28 \%$ patients, genital warts were seen in 65 patients, molluscum contagiosum (Fig. 3) was seen in 5\% patients and syphilis was seen in $4 \%$ patients. It was seen that the commonest symptom was pruritis seen in $46 \%$ patients and pain was seen in $4 \%$ patients. Discharge per vaginum was seen in $28 \%$ patients and the discharge was candidal in $20 \%$ patients, trichomonal in $6 \%$ patients and the cause was bacterial vaginosis seen in $2 \%$ patients.

Many of the symptoms and signs are so common that they are not usually considered as being abnormal, but regarded as physiological and can sometimes provide contributory evidence of pregnancy [6]. 


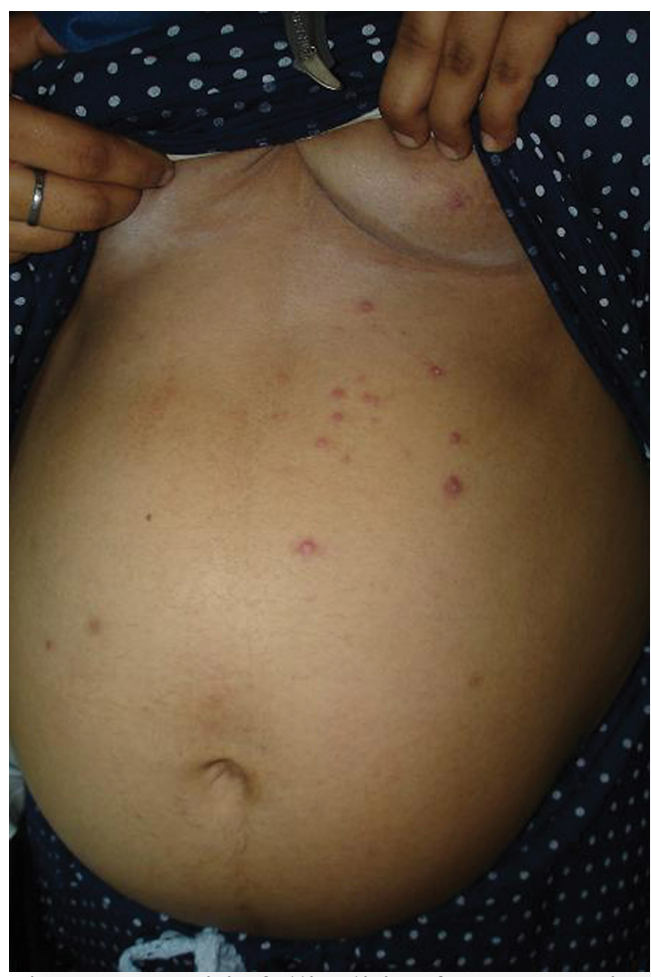

Figure 1. Pruritic folliculitis of pregnancy in a seventh month pregnant patient

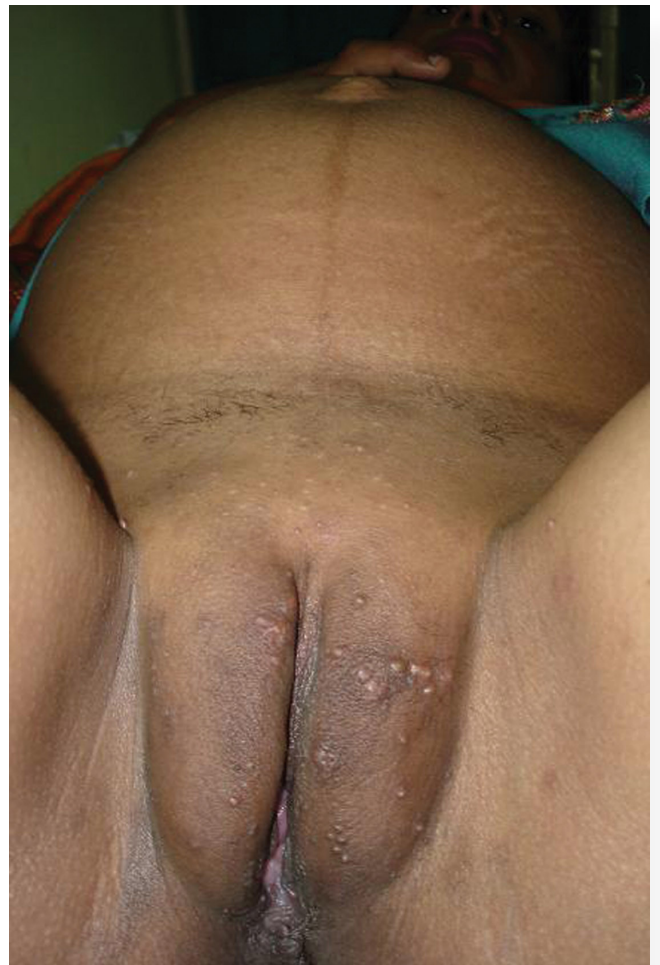

Figure 3. Genital MC in a eighth month pregnant patient

The commonly encountered physiological changes include striae distensae (occurring in up to $90 \%$ of pregnant women), hormonal alterations resulting in melasma (occurring in up to $75 \%$ of women during pregnancy) and generalized hyperpigmentation $[7,8]$. Vascular alterations result in edema, palmar erythema, spider nevi, varicosities, cutis marmorata, gingival edema and redness. Similarly the activity of eccrine and sebaceous glands increases, while that of apocrine gland

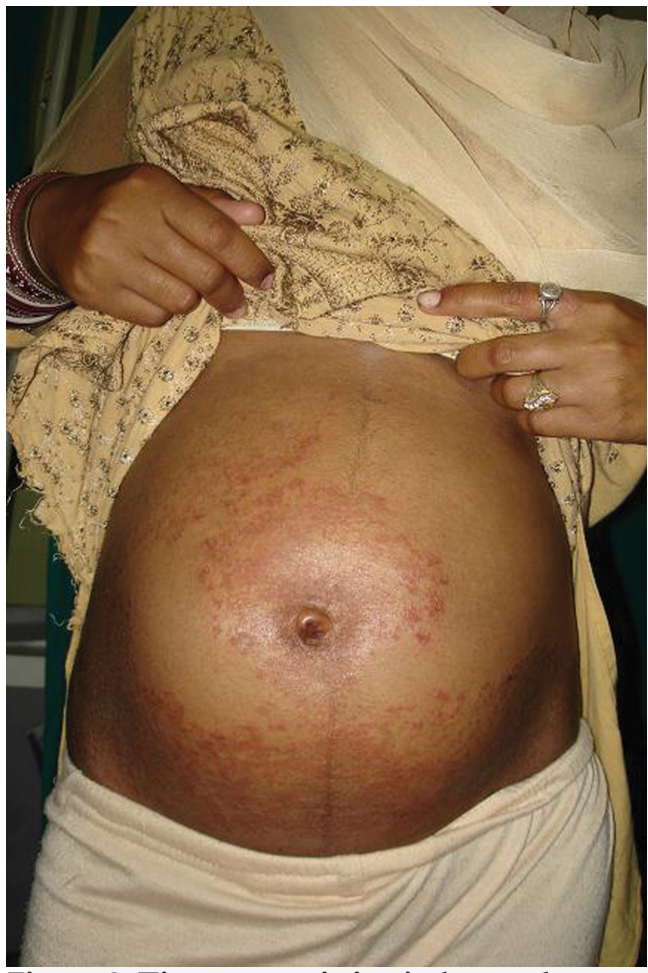

Figure 2. Tinea corporis in sixth month pregnant patient

decreases. In addition, pregnancy can modify a number of concomitant dermatoses and there are some pathological skin conditions that are virtually pregnancy specific. The most common physiological changes are pigmentary alterations, stretch marks, vascular spiders and telogen effluvium [9].

Pruritic urticarial papules and plaques of pregnancy (PUPPP) is the most common dermatosis of pregnancy, and is also known as toxemic rash or polymorphic eruption of pregnancy. Pruritic urticarial papules and plaques occur in 1 of 160 to 240 pregnancies, and are more common in white women [10]. Classically, this disease occurs in primigravidas during the third trimester, and the incidence is higher in multiple gestations (ie, $0.5 \%$ of single births, $2.9 \%$ of twin pregnancies, and $14 \%$ of triplets) [11]. Pruritic urticarial papules and plaques most commonly present as intensely pruritic papules within striae distensae. Over the next several days, erythematous, urticarial papules and plaques spread to involve the trunk and extremities $[12,13]$. The periumbilical region, face, palms, and soles are usually spared. Occasionally vesicles, purpura, targetoid, eczematous, or polycyclic lesions are seen. A relationship to skin distension has been proposed due to the higher prevalence of PUPPP in multiple gestations and in women with increased weight gain during pregnancy, or the condition may represent a cutaneous response to the presence of circulating fetal cells that have invaded maternal skin $[14,15]$.

Pemphigoid gestationis (PG), also known as herpes gestationis (HG), is an autoimmune bullous disease of pregnancy. Despite its name, this disease has no relationship to herpes simplex virus, but was so called because of the herpes-like nature of the blisters [16]. This dermatosis classically develops in the second or third trimester (mean of 21 weeks) [17]. 
It occurs in 1 in 50,000 pregnancies, and has a strong association with HLA-DR3 and HLA-DR4, which might explain the greater prevalence of this condition in white women compared to black women [18]. Pemphigoid gestationis begins with the sudden onset of intensely itchy, urticarial lesions, which are found on the abdomen in 50\% of cases. At this stage, it is very difficult to distinguish this disease from PUPPP. The lesions then progress to a generalized bullous eruption that usually spares the face, mucous membranes, palms and soles [19]. The disease often resolves during the latter part of pregnancy, and flares at delivery or immediately postpartum in more than $60 \%$ of cases; $25 \%$ of cases appear for the first time after delivery [20]. Biopsy results reveal a subepidermal blister with an eosinophil-predominant infiltrate. The infiltrate is localized to the dermal-epidermal junction and perivascular areas [21]. Direct Immunofluorescence (DIF) is the most sensitive and specific assay for differentiating PG from PUPPP. Performed on perilesional skin, DIF shows a linear band of C3 and/ or IgG at the basement membrane. Salt-split skin studies demonstrate antibody binding to the roof of the vesicle [22].

Intrahepatic cholestasis of pregnancy (ICP) is caused by maternal intrahepatic bile secretory dysfunction. A genetic predisposition for this disorder has been described. This disease is characterized by intense generalized pruritus that usually begins in the third trimester. Although constant, the pruritus is classically much worse at night. It may be most severe on the palms and soles. The important feature of intrahepatic cholestasis is the absence of primary lesions, such that excoriations are the only cutaneous finding [23]. Jaundice is present in a minority of patients. Symptoms tend to dissipate within days of delivery, but there is a tendency toward later development of gallbladder disease in these women. There is a potential for recurrence in subsequent pregnancies or with OC use. Fetal risk is also a matter of concern.

Pruritic folliculitis of pregnancy (PFP) classically occurs in the second and third trimester [24]. Women present with generalized red, follicularly based papules. These are distributed on the chest and back in most cases, and resemble the monomorphic acne that may occur in women who are taking oral corticosteroids. The lesions are variably pruritic. Although the pathogenesis is unknown, many consider PFP to be a form of steroid acne. There is no evidence of immunologic or hormonal abnormalities in this condition. Interestingly, there is an overall preponderance of male infants (2:1) delivered to women with this condition [25]. Histologically, the condition is characterized by sterile folliculitis. Features include acute folliculitis with mixed inflammatory cells, upper dermal edema, spongiosis, and a negative Gram stain. Direct immunofluorescence is negative.

Prurigo of pregnancy (PP) is the most poorly characterized gestational dermatosis. It has also been described as Besnier's prurigo of pregnancy and papular dermatitis of Spangler. Prurigo of pregnancy is a diagnosis of exclusion, and a large number of pruritic entities unrelated to pregnancy must be considered. It classically occurs in the second half of pregnancy, affecting about 1 in 300 pregnant women [26]. This condition presents clinically as discrete, bite-like papules on the extremities that resemble scabies or other arthropod bites. The lesions consist of erythematous papules and nodules on the extensor surfaces of extremities and occasionally the abdomen [27]. Prurigo of pregnancy is often seen in women with an atopic background, and is associated with increased serum levels of immunoglobulin E (IgE)-supporting the notion that PP may represent a gestational variant of atopic dermatitis occurring as a result of common pruritus gravidarum [28]. Liver function studies, including serum bile acids, should be performed to rule out cholestasis or hepatitis. At a minimum, the differential diagnosis also should include an allergic reaction and scabies or other infestation. The histopathology of PP is nonspecific, usually demonstrating a chronic, inflammatory cell infiltrate with superficial excoriation. Direct immunofluorescence is negative. Treatment is symptomatic, including topical emollients, topical midpotency corticosteroids, and systemic antihistamines. There is no associated maternal or fetal risk. The eruption typically resolves soon after delivery.

Approximately two million pregnant women are affected by sexually transmitted diseases or STDs during each year in the United States. Pregnancy offers no protection against sexually transmitted diseases leaving pregnant women vulnerable to the same STDs as women who are not pregnant. Sexually transmitted diseases can cause devastating consequences women who are not pregnant; the consequences of sexually transmitted diseases can be significantly more dangerous for pregnant women [29]. The occurrence of genital warts while pregnant can be a foremost basis of apprehension for mothers to be. It puts the unborn child at risk of getting the infection. There is also a chance that genital warts can be passed along to newborn babies through a contaminated birth canal. The main concern is that the unborn baby of a mother infected with genital warts may contract laryngeal papillomatosis, which is a life-threatening condition.

\section{Conclusions}

Pregnant women are prone to suffer from a wide range of dermatological problems and sexually transmitted diseases, apart from the specific dermatoses of pregnancy. This study emphasises the need for a scrupulous and meticulous search for dermatological and sexually transmitted diseases instead of a casual cursory examination and dismissing the patients with symptoms attributing them to the normal course of pregnancy. These pruritic dermatoses are unique to the gravid state. A detailed history and awareness of clinical presentation facilitate confirmation of the diagnosis, and will direct the most appropriate laboratory evaluation in an effort to minimize maternal and fetal morbidity. In addition, monitoring of liver function deserves special consideration.

\section{REFERENCES}

1. Muzaffar F, Hussain I, Haroon TS: Physiologic skin changes during pregnancy: A study of 140 cases Int J Dermatol. 1998;37:42931.

2. Martin AG, Leal-Khouri S: Physiological skin changes associated with pregnancy. Int J Dermatol. 1992;31:375-8.

3. Winton GB, Lewis CW: Dermatoses of pregnancy. J Am Acad Dermatol. 1982;6:977-98. 
4. Roger D, Vaillant L, Fignon A, Pierre F, Bacq Y, Brechot JF, et al: Specific pruritic dermatoses of pregnancy: A prospective study of 3192 women. Arch Dermatol. 1994;130:734-9.

5. Ambros-Rudolph CM, Müllegger RR, Vaughan-Jones SA, Kerl H, Black MM: The specific dermatoses of pregnancy revisited and reclassified: results of a retrospective two-center study on 505 pregnant patients. J Am Acad Dermatol. 2006;54:395.

6. Kroumpouzos G, Cohen LM: Dermatoses of pregnancy. J Am Acad Dermatol. 2001;45:1-19.

7. Ruiz-Villaverde R, Blasco Melguizo J, Naranjo-Sintes R: Pigmentary demarcation lines in a pregnant Caucasian woman. Int J Dermatol. 2004;43:911-2.

8. Shornick JK: Dermatoses of pregnancy. Semin Cutan Med Surg. 1998; 17:172.

9. Esteve E, Saudeau L, Pierre F, Barruet K, Vaillant L, Lorette G: Physiological cutaneous signs in normal pregnancy: A study of 60 pregnant women. Ann Dermatol Venereol. 1994;121:227-31.

10. Aronson IK, Bond S, Fiedler VC, Vomvouras S, Gruber D, Ruiz C: Pruritic urticarial papules and plaques of pregnancy: Clinical and immunopathologic observations in 57 patients. J Am Acad Dermatol. 1998;39:9-13.

11. Elling SV, McKenna P, Powell FC: Pruritic urticarial papules and plaques of pregnancy in twin and triplet pregnancies. J Eur Acad Dermatol Venereol. 2000;14:378.

12. Beckett MA, Goldberg NS: Pruritic urticarial plaques and papules of pregnancy and skin distention. Arch Dermatol. 1991;127:125.

13. Aractingi S, Berkane N, Bertheau P, Le Goué C, Dausset J, Uzan $\mathrm{S}$, et al: Fetal DNA in skin of polymorphic eruptions of pregnancy. Lancet. 1998;352:1898.

14. Beltrani VP, Beltrani VS: Pruritic urticarial papules and plaques of pregnancy: a severe case requiring early delivery for relief of symptoms. J Am Acad Dermatol. 1992;26:266.

15. Regnier S, Fermand V, Levy P, Uzan S, Aractingi S: A casecontrol study of polymorphic eruption of pregnancy. J Am Acad Dermatol 2008; 58:63Winton GB. Skin diseases aggravated by pregnancy. J Am Acad Dermatol. 1989;20:1-13.

16. Jenkins RE, Jones SA, Black MM: Conversion of pemphigoid gestationis to bullous pemphigoid-two refractory cases highlighting this association. Br J Dermatol. 1996;135:595.
17. Jenkins RE, Hern S, Black MM: Clinical features and management of 87 patients with pemphigoid gestationis. Clin Exp Dermatol. 1999;24:255.

18. Chi CC, Wang SH, Charles-Holmes R, Ambros-Rudolph C, Powell J, Jenkins R, et al: Pemphigoid gestationis: early onset and blister formation are associated with adverse pregnancy outcomes. Br J Dermatol. 2009;160:12-22.

19. Shimanovich I, Skrobek C, Rose C, Nie Z, Hashimoto T, Bröcker EB, et al: Pemphigoid gestationis with predominant involvement of oral mucous membranes and IgA autoantibodies targeting the C-terminus of BP180. J Am Acad Dermatol. 2002;47:780.

20. Aoyama Y, Asai K, Hioki K, Funato M, Kondo N, Kitajima $\mathrm{Y}$ : Herpes gestationis in a mother and newborn: immunoclinical perspectives based on a weekly follow-up of the enzyme-linked immunosorbent assay index of a bullous pemphigoid antigen noncollagenous domain. Arch Dermatol. 2007;143:1168.

21. Shimanovich I, Brocker EB, Zillikens D: Pemphigoid gestationis: new insights into the pathogenesis lead to novel diagnostic tools. BJOG. 2002;109:970.

22. Di Zenzo G, Calabresi V, Grosso F, Caproni M, Ruffelli M, Zambruno G: The intracellular and extracellular domains of BP180 antigen comprise novel epitopes targeted by pemphigoid gestationis autoantibodies. J Invest Dermatol. 2007;127:864.

23. Wilson B, Haverkamp A: Cholestatic jaundice of pregnancy: new perspectives. Obstet Gynecol. 1979;54:650-652.

24. Wilkinson SM, Buckler H, Wilkinson N, O'Driscoll J, Roberts MM: Androgen levels in pruritic folliculitis of pregnancy. Clin Exp Dermatol. 1995;20:234-6.

25. Kroumpouzos G, Cohen LM: Pruritic folliculitis of pregnancy. J Am Acad Dermatol. 2000;43:132.

26. Black MM: Prurigo of pregnancy, papular dermatitis of pregnancy and pruritic folliculitis of pregnancy. Semin Dermatol. 1989;8:23-5.

27. Vaughan Jones SA, Black MM: Pregnancy dermatoses. J Am Acad Dermatol. 1999;40:233.

28. Kroumpouzos G, Cohen LM: Dermatoses of pregnancy. J Am Acad Dermatol. 2001;45:1.

29. Piot P, Laga M: Genital ulcers, other sexually transmitted diseases and the sexual transmission of HIV. Br Med J. 1989;298:623-4.

Copyright by Neerja Puri, et al. This is an open access article distributed under the terms of the Creative Commons Attribution License, which permits unrestricted use, distribution, and reproduction in any medium, provided the original author and source are credited. 